\title{
ON THE EXISTENCE OF FUNDAMENTAL SOLUTIONS OF BOUNDARY PROBLEMS
}

\section{J. BARROS-NETO ${ }^{1}$}

In this paper we prove an existence theorem for fundamental solutions (see definition below) of a large class of boundary value problems in a half space which includes the Cauchy problem for hyperbolic and parabolic operators with constant coefficients.

As a particular case, we obtain Shilov's result [4] on the existence of Green's kernels (see definition below) of Cauchy problems.

The technique employed is that of Fourier transform of tempered distributions. Our theorem relies on Hörmander's result on the division of a tempered distribution by a polynomial [3].

The result of this paper is related to our previous results [1] on the existence of fundamental kernels for regular elliptic boundary problems.

1. Let $P\left(D, D_{t}\right)$ where $D=\left(D_{1}, \cdots, D_{n}\right), D_{j}=(1 / i)\left(\partial / \partial x_{j}\right)$ and $D_{t}=(1 / i)\left(\partial / \partial x_{t}\right)$ be a partial differential operator with constant coefficients. Let $P(\xi, \tau)$ be its characteristic polynomial, assume that the highest order coefficient of $\tau$ is independent of $\xi$ and that all the roots of the equation in $\tau$

$$
P(\xi, \tau)=0
$$

have imaginary parts bounded below by a constant $C$, for all $\xi \in R^{n}$.

If $m$ is the degree of $P(\xi, \tau)$ in $\tau$, let

$$
Q_{1}\left(D, D_{t}\right), \cdots, Q_{m}\left(D, D_{t}\right)
$$

be $m$ given partial differential operators with constant coefficients. The operators

$$
\left(P\left(D, D_{t}\right), Q_{1}\left(D, D_{t}\right), \cdots, Q_{m}\left(D, D_{t}\right)\right)
$$

define a boundary problem in the half space

$$
R_{+}^{n+1}=\left\{(x, t): x \in R^{n}, t>0\right\} .
$$

THEOREM. Under the above conditions, there are $m$ tempered distributions

$$
K_{j}(x, t) \in S^{\prime}\left(R^{n}\right), \quad 1 \leqq j \leqq m,
$$

Received by the editors June 2, 1969.

1 This research was supported by N.S.F. grant GP-11845. 
depending upon a parameter $t>0$, verifying the boundary problem

$$
\begin{aligned}
& P\left(D, D_{t}\right) K_{j}(x, t)=0 \quad \text { on } \quad R_{+}^{n+1} \\
& \lim _{l \rightarrow 0+} Q_{l}\left(D, D_{t}\right) K_{j}(x, t)=\delta_{l, j} \cdot \delta, \quad 1 \leqq j \leqq m,
\end{aligned}
$$

where $\delta_{l, j}$ is the Kronecker symbol, $\delta$ the Dirac measure in $R^{n}$ and the limit is taken in $S^{\prime}\left(R^{n}\right)$.

Proof. 1. Fix $\xi$ in $R^{n}$ and let

$$
\tau_{1}=\tau_{1}(\xi), \cdots, \tau_{m}=\tau_{m}(\xi)
$$

be the $m$ roots (counting multiplicities) of

$$
P(\xi, \tau)=0 .
$$

Let $f_{1}, \cdots, f_{m}$ be analytic functions of a complex variable $\tau$ and define

$$
R\left(P, f_{1}, \cdots, f_{m}\right)=\operatorname{det} f_{j}\left(\tau_{l}\right) / \prod_{k<l}\left(\tau_{l}-\tau_{k}\right) .
$$

It can be shown $[2$, pp. 231,232$]$ that $R\left(P, f_{1}, \cdots, f_{m}\right)$ is defined even in the case of multiple zeros, it is an analytic function of all the variables $\tau_{l}$ and we have the estimate

$$
\left|R\left(P, f_{1}, \cdots, f_{m}\right)\right| \leqq \prod_{j=1}^{m}\left(\sum_{k=0}^{m-1} \sup _{z \in K} \frac{\left|f_{j}^{(k)}(z)\right|}{j !}\right)
$$

where $K$ denotes the convex hull of the zeros $\tau_{1}, \cdots, \tau_{m}$ of $P$.

2. Set $f_{j}(\tau)=Q_{j}(\xi, \tau)$ and define

$$
\begin{array}{r}
C(\xi)=R\left(P, Q_{1}, \cdots, Q_{m}\right)=\operatorname{det} Q_{j}\left(\xi, \tau_{l}(\xi)\right) / \prod_{k<l}\left(\tau_{l}(\xi)-\tau_{k}(\xi)\right), \\
\xi \in R^{n},
\end{array}
$$

which is called the characteristic function of the given boundary problem.

$C(\xi)$ is, obviously, a symmetric function of $\tau_{1}, \cdots, \tau_{m}$, thus it can be expressed as a polynomial on the coefficients of $\tau$ in $P(\xi, \tau)$, that is to say, $C(\xi)$ is a polynomial in $\xi$.

3. Consider, now, the following function

$$
H_{j}(\xi, t)=R\left(P, Q_{1}(\xi, \tau(\xi)), \cdots, e_{j}^{i t \tau(\xi)}, \cdots, Q_{m}(\xi, \tau(\xi))\right),
$$

$1 \leqq j \leqq m$, defined for all $\xi \in R^{n}$ and all $t \geqq 0$.

From our assumption on $P(\xi, \tau)$ it follows that there are constants $A$ and $B$ such that the zeros of $P(\xi, \tau)$ satisfy the inequality 


$$
|\tau| \leqq A\left(|\xi|^{B}+1\right) .
$$

From inequalities (2), (3) and our assumption that the imaginary parts of the roots of $P(\xi, \tau)=0$ are bounded below by $C$ we get for each fixed $t \geqq 0$, the estimate

$$
\left|H_{j}(\xi, t)\right| \leqq A^{\prime}|\xi|^{B^{\prime}} e^{-t C}, \quad \xi \in R^{n} .
$$

This shows that, for each $t \geqq 0, H_{j}(\xi, t)$ defines a tempered distribution in $\xi$.

4. According to a result due to Hörmander [3], we can divide the tempered distribution $H_{j}(\xi, t)$ by the polynomial $C(\xi)$ and the result

$$
U_{j}(\xi, t)=H_{j}(\xi, t) / C(\xi), \quad 1 \leqq j \leqq m,
$$

is a tempered distribution on $R^{n}$, depending upon $t \geqq 0$.

Next, it can be proved that each $U_{j}(\xi, t)$ verifies the following initial valued problem derived from (1) by taking Fourier transform on $x$ :

$$
\begin{array}{rlrl}
P\left(\xi, D_{t}\right) U_{j}(\xi, t) & =0, & \forall \xi \in R^{n}, \quad \forall t \geqq 0, \\
Q_{l}\left(\xi, D_{t}\right) U_{j}(\xi, 0) & =\delta_{j, l}, \quad l=1,2, \cdots, m .
\end{array}
$$

5. Let

$$
K_{j}(x, t)=F_{\xi}^{-1} U_{j}(\xi, t), \quad 1 \leqq j \leqq m,
$$

be the inverse Fourier transform of $U_{j}(\xi, t)$. For each $t \geqq 0, K_{j}(x, t)$ $\in S^{\prime}\left(R^{n}\right)$ and it is obvious that $K_{j}$ verifies problem (1). Q.E.D.

Definition. We call $\left(K_{1}, K_{2}, \cdots, K_{m}\right)$ the fundamental solution of the boundary problem $\left(P, Q_{1}, \cdots, Q_{m}\right)$.

A solution of the boundary value problem

$$
\begin{aligned}
P\left(D, D_{t}\right) u & =0 \quad \text { in } \quad R_{+}^{n+1}, \\
\left.Q_{j}\left(D, D_{t}\right) u\right|_{t=0} & =g_{j}, \quad 1 \leqq j \leqq m,
\end{aligned}
$$

where $g_{j}$ are smooth functions is given by

$$
u(x, t)=\sum_{j=1}^{m} K_{j}(\cdot, t) * g_{j},
$$

the convolution being taken with respect to $x$.

Application. If we take as boundary operators the following ones

$$
Q_{1}=1, \quad Q_{2}=D_{t}, \cdots, Q_{m}=D_{t}^{m-1}
$$

our theorem gives, as a particular case, the Green's kernel of the 
Cauchy problem, namely, a tempered distribution

$$
G(x, t) \in S^{\prime}\left(R^{n}\right), \quad t \geqq 0,
$$

verifying the equations:

$$
\begin{aligned}
& P\left(D, D_{t}\right) G(x, t)=0 \text { in } R_{+}^{n+1}, \\
& G(x, 0)=0 \\
& D_{t} G(x, 0)=0 \\
& \ldots \ldots \ldots \ldots \cdots \cdots \\
& D_{t}^{m-1} G(x, 0)=\delta . \\
& \text { REFERENCES }
\end{aligned}
$$

1. J. Barros-Neto, Kernels associated to general elliptic problems, J. Functional Anal. 3 (1969), 173-192.

2. L. Hörmander, On the regularity of the solutions of boundary problems, Acta Math. 99 (1958), 225-264. MR 24, A1503.

3. - On the division of distributions by polynomials, Ark. Math. 3 (1958), 555-568. MR 23 \#A2044.

4. G. E. Silov, Well-posed boundary-value problems in a half-space for linear partial differential equations with constant coefficients, Uspehi Mat. Nauk. 19 (1964), no. 3 (117), 3-52 = Russian Math. Surveys 19 (1964), no. 3, 1-52. MR 29 \#4988.

RUTGERS UNIVERSITY 